\title{
Methanogenesis Coupled Bioremediation of Hydrocarbon Contaminated Soil and Groundwater
}

\author{
Krisztián Laczi ${ }^{1}$, Jacob Manyiwa Shume ${ }^{1}$, Attila Bodor ${ }^{2}$, Naila Bounedjoum ${ }^{1}$, György Erik Vincze ${ }^{1}$, \\ Katalin Perei ${ }^{1}$, Tamás Kovács ${ }^{3}$, Gábor Rákhely ${ }^{1,2}$ \\ ${ }^{1}$ Department of Biotechnology, University of Szeged, \\ 52. Közép fasor, Szeged, H-6726, Hungary \\ ${ }^{2}$ Institute of Biophysics, Biological Research Centre, \\ 62. Temesvári krt. Szeged, H-6726, Hungary \\ ${ }^{3}$ Department of Biotechnology, Nanophagetherapy Center, Enviroinvest Corp., \\ 2. Kertváros u., Pécs, H-7632, Hungary \\ rakhely@brc.hu
}

\section{Extended Abstract}

At the moment, the gobal economy is based on petrochemical products; hence hydrocarbons are among the main pollutants all over the world. Hydrocarbon contaminations pose a serious threat to the environment and human health. Therefore, removal of such pollutants is among the top evironmental challenges. A number of biotechnologies have been develepod for aerobic oxidation of various hydrocarbons, however, remediation under anoxic conditions such as in the deep soil layers is still a bigg challenge requiring economic solutions. The innate microflora might have the metabolic capacity to utilize hydrocarbons as a carbon and energy source. However, under anaerobic conditions, alternative, expensive electron acceptors are required, or in their absence, a portion of the mineralized hydrocarbons can be converted into methane, thus can be collected as an energy carrier and can decrease the costs of remediation.

In this study, we investigated an area which has been used as an army airport in the last. The remediation of the area has already been attempted but - likely due to the groundwater flows - the pollution appeared again. The area was remonitored: samples (12) were taken upto the groundwater level $(7.5-8 \mathrm{~m})$ and were chemically and microbiologically characterized. Metagenomic analyses revealed that the most abundant genera were Smithella (16\%) alongside with Rhodoferax (9\%). The members of these two genera were recognized as alkane [1] and aromatics [2] degraders respectively. Methanosaeta (3\%) and Methanoregula (6\%) were also found among the most abundant genera. Applying a binning method, the genomes belonging to the genera above were recovered with the exception of Methanosaeta. In the reconstructed genome of Smithella sp., we identified an alkylsuccinate synthase gene that participates in the activation of aliphatic hydrocarbons under anaerobic conditions [3]. Genes participating in the degradation of aromatic compounds were also found in the Rhodoferax genome. Batch fermentation experiments showed that the enrichment culture derived from this soil sample was able to convert hydrocarbons into methane, therefore it can be used for microbial enhanced energy recovery.

\section{Acknowledgements} 00010).

The project was supported by the European Union and the Hungarian State (grant agreement No.: EFOP-3.6.2-16-2017-

\section{References}

[1] B. Tan, C. Nesbø, and J. Foght,"Re-analysis of omics data indicates Smithella may degrade alkanes by addition to fumarate under methanogenic conditions,” The ISME Journal, vol. 8, no. 12, pp. 2353-2356, 2014.

[2] C. Risso, J. Sun, K. Zhuang, R. Mahadevan, R. DeBoy, W. Ismail, S. Shrivastava, H. Huot, S. Kothari, S. Daugherty, O. Bui, C.H. Schilling, D.R. Lovley, and B.A. Methé,"Genome-scale comparison and constraint-based metabolic reconstruction of the facultative anaerobic Fe(III)-reducer Rhodoferax ferrireducens," BMC Genomics, vol. 10, pp. 447, 2009. 
[3] A. V. Callaghan, I. A. Davidova, K. Savage-Ashlock, V. A. Parisi, L. M. Gieg, J. M. Suflita, J. J. Kukor, and B. Wawrik, "Diversity of benzyl- and alkylsuccinate synthase genes in hydrocarbon-impacted environments and enrichment cultures," Environmental Science \& Technology, vol. 44, no. 19, pp. 7287-7294, 2010. 